\title{
Barley yellow dwarf virus Can Be Inoculated During Brief Intracellular Punctures in Phloem Cells Before the Sieve Element Continuous Salivation Phase
}

\author{
Jaime Jiménez, † María Arias-Martín, Aránzazu Moreno, Elisa Garzo, and Alberto Fereres \\ Instituto de Ciencias Agrarias, Consejo Superior de Investigaciones Científicas, 28006 Madrid, Spain \\ Accepted for publication 10 October 2019.
}

\begin{abstract}
The distinguished intracellular stylet puncture called phloem-pd (potential drop [pd]) produced by Myzus persicae has been associated with the transmission of the semipersistently transmitted, phloemlimited Beet yellows virus (BYV, Closterovirus). However, the production of intracellular punctures in phloem cells (phloem-pd) by other aphid species and their role in the transmission of persistently transmitted, phloem-limited viruses are still unknown. Previous studies revealed that inoculation of the persistently transmitted, phloemlimited Barley yellow dwarf virus (BYDV, Luteovirus) is associated mainly with the sieve element continuous salivation phase (E1 waveform). However, the role of brief intracellular punctures that occur before the E1 phase in the inoculation of BYDV by aphids is unknown. We aimed to investigate whether the bird cherry-oat aphid Rhopalosiphum padi (Hemiptera: Aphididae) produced a stereotypical phloem-pd and to study its role in the inoculation of BYDV. The feeding
\end{abstract}

ABSTRACT

The study of aphid stylet activities in plant in relation to transmission of plant viruses has been paramount in the latest studies of plant virus transmission (Fereres 2016; Fereres and Moreno 2009). Since the first study by Scheller and Shukle (1986), several works have focused on associating specific aphid stylet activities with successful virus transmission after interrupting the feeding process of the aphid (Jiménez et al. 2018; Martín et al. 1997; Prado and Tjallingii 1994). The electrical penetration graph (EPG) technique (Tjallingii 1978) has been key in studying the aphid feeding process in association with the transmission of plant viruses. Hence, different activities of the aphid stylets in plant tissues have been associated to specific EPG waveforms determined by specific patterns or changes in electrical potential. Aphid stylet activities associated with plant tissues begin with intercellular penetration through the epidermis and mesophyll cells (stylet pathway phase, waveform C), which is interrupted by intermittent, very brief intracellular punctures into nonvascular cells, which are reflected by a voltage drop (potential drop [pd]) (Tjallingii 1985, 1988), and previously referred to as standard potential drops (standard-pds) (Chen et al. 1997). After numerous and consecutive pds, aphids often reach the phloem tissues, and they start the sieve element continuous salivation phase (E1 waveform) followed by subsequent phloem sap ingestion (E2 waveform) (Prado and Tjallingii 1994).

†Corresponding author: J. Jiménez; jaime.jimenez@ufl.edu

Funding: This work was funded by the Ministerio de Economia y Competitividad grant AGL2013-47603-C2-2-R (BES-2014-067806) and the Consejo Superior de Investigaciones Cientificas.

The author(s) declare no conflict of interest.

(c) 2020 The American Phytopathological Society behavior of viruliferous $R$. padi individuals in barley (Hordeum vulgare) was monitored via the electrical penetration graph (EPG) technique. The feeding process was artificially terminated after the observation of specific EPG waveforms: standard-pds, phloem-pd, and E1. Analysis of the EPG recordings revealed the production of a phloem-pd pattern by $R$. padi, in addition to a short, distinct E1-like pattern (short-E1), both resulting in successful inoculation of BYDV. Also, the transmission efficiency of BYDV was directly proportional to the time spent by aphids in intracellular salivation in phloem cells. Finally, we discussed the main differences between the inoculation process of semipersistent and persistently transmitted phloem-limited viruses by aphids.

Keywords: aphid probing behavior, electrical penetration graphs, phloempd, Rhopalosiphum padi, short-E1

Insect-transmitted viruses are classified in two groups depending on their persistence within the vector: noncirculative (NC) and circulative viruses (CV) (Blanc and Michalakis 2016; Fereres and Raccah 2015). Also, within NC category, two groups are distinguished: nonpersistent (NP) and semipersistent (SP) transmitted viruses (Mauck et al. 2018). The specific aphid stylets activities related to successful NP virus transmission have been accurately described, with the standard-pds occurring in the epidermis and mesophyll cells as responsible for their inoculation and acquisition. Whereas inoculation is associated with the subphase II- 1 of the pd (injection of watery saliva into the cell) (Powell 2005), virus acquisition takes place during the subphase II-3 (ingestion of cell cytoplasm) (Martín et al. 1997; Powell et al. 1995). The transmission mechanism of SP viruses depends on their localization in specific host plant tissues. For instance, for SP viruses located in all plant tissues (e.g., Cauliflower mosaic virus (CaMV), Caulimovirus), inoculation may occur during the first intracellular punctures, although the inoculation mechanism seems to be different from that of NP viruses (Moreno et al. 2012). With regard to the acquisition process, CaMV is preferentially acquired from the phloem tissues (Palacios et al. 2002). However, other SP viruses, such as Beet yellows virus (BYV, Closterovirus), are limited to the phloem cells in the infected plants (Esau 1968). Whereas acquisition of BYV is associated with long periods (hours) of phloem sap ingestion by Myzus persicae, inoculation occurs at the highest rate after brief intracellular penetrations produced by the aphid into the phloem tissues (called phloem-pds; Jiménez et al. 2018), more precisely in the companion (CC) and sieve element (SE) cells (Jiménez et al. 2019).

Whereas the majority of persistently and semipersistently aphidtransmitted viruses are limited to the phloem cells, their interactions with their insect vectors and their mechanism of inoculation may be different. Barley yellow dwarf virus (BYDV, Luteovirus) is the type member of the Luteoviridae family, and it has been thoroughly studied. BYDV follows a circulative-nonpropagative virus 
transmission mode (Gray and Gildow 2003), and it is one of the most important virus diseases in cereal crops and grasses worldwide, infecting $>150$ species in the Poaceae family (D'Arcy 1995). Moreover, many Poaceae species still need to be studied for their ability to be potential hosts of BYDV because new species are continuously identified as hosts (Ingwell et al. 2014; Ingwell and Bosque-Pérez 2015). BYDV primary infection causes stunted growth and yellow coloration of leaves, leading to a collapse of sieve elements, resulting in necrosis, also observed in CCs and phloem parenchyma cells (Esau 1957). Twenty-five aphid species have been reported to transmit BYDV (Halbert and Voegtlin 1995), with the bird cherry-oat Rhopalosiphum padi (L.) (Hemiptera: Aphididae) and the English grain aphid Sitobion avenae (Fabricius) (Hemiptera: Aphididae) as the main vectors (Gildow 1990; Habekuß et al. 2002). During acquisition, BYDV particles circulate through the vector body and pass the intestinal cells to the hemocoel, circulating toward the accessory salivary glands (Gildow 1999). Approximately 12 to $16 \mathrm{~h}$ is needed for luteoviruses to be actively transported through the gut epithelial cell and released into the aphid hemocoel (Garret et al. 1996). Virus particles survive in the hemolymph for several weeks and are passively transported to the accessory salivary glands (Gildow and Gray 1993; Gray and Gildow 2003) to be injected with the saliva after aphid stylet intrusion into the phloem sieve elements of the host plant (Prado and Tjallingii 1994).

Several works have focused on the transmission of BYDV by monitoring the aphid probing behavior of the two main aphid vector species: $R$. padi and S. avenae. Early work by Scheller and Shukle (1986) using an alternating current EPG device showed that the inoculation of BYDV increased as the number of aphid stylets penetrations in phloem increased. Primary BYDV inoculation was obtained during the so-called X-waveform, an EPG pattern associated with the contact of aphid stylets with phloem cells before sap ingestion from the phloem (ingestion phase [Ip]). Some years later, aphid stylet activities involved in BYDV transmission were also studied for $R$. padi with a direct current EPG device, with virus transmission associated mainly with waveforms E1 (inoculation) and E2 (acquisition) (Prado and Tjallingii 1994). However, the same authors observed that inoculation occurred, at a very low rate, during EPG recordings that were interrupted before the sieve element continuous salivation phase (E1 waveform) (Prado and Tjallingii 1994).

Therefore, the goal of our work was to understand the mechanism of inoculation of BYDV by $R$. padi and to investigate whether the recently described phloem-pd activity is involved in the inoculation of the virus. Therefore, EPG recordings were artificially terminated after specific EPG patterns were observed, including the phloem-pd recently described for $M$. persicae and BYV transmission (Jiménez et al. 2018). Furthermore, we studied the inoculation process of BYDV by relating inoculation efficiency with the duration of intracellular stylet insertions of $R$. padi in phloem cells. Finally, we compared and discussed the main differences between the inoculation process of a circulative, persistent virus (BYDV) and a noncirculative semipersistent virus (BYV).

\section{MATERIALS AND METHODS}

Plants, aphids, and virus maintenance. Experiments were conducted in barley plants (Hordeum vulgare 'Shakira') at a single unfolded leaf stage (growing stage 11, Zadoks growth scale). Test plants for EPG experiments were maintained in an insect-free growth chamber at a temperature of $24: 20^{\circ} \mathrm{C}$ (day:night) and a photoperiod of 16:8 h (day:night).

Viruliferous, apterous $R$. padi (L.) young adults reared in BYDV-infected $H$. vulgare were used to run the EPG recordings. BYDV source barley plants were inoculated by placing a group of 10 viruliferous, apterous $R$. padi on a barley plant at phenological growing stage 11 and were renewed after 4 to 5 weeks. The virus isolate used was BYDV-PAV serotype, kindly provided by Antje Habekuß (Institute for Resistance and Stress Tolerance, Quedlinburg, Germany). Our initial nonviruliferous $R$. padi colony was generated from a single virginoparous female collected from a barley plant in the experimental field La Poveda (Madrid, Spain) in 2013. Viruliferous aphid colonies were weekly checked to remove $R$. padi individuals to avoid overcrowding. The viruliferous colony was maintained in a growth chamber at a temperature of $24: 20^{\circ} \mathrm{C}$ (day:night) and a photoperiod of 16:8 h (day:night).

Virus infection in both test and source plants was doubly checked by first conducting a visual inspection of symptoms with later serological detection by double-antibody sandwich enzyme-linked immunosorbent assay test (Clark and Adams 1977) 4 weeks after virus inoculation. After aphid infestation, plants were sprayed with an imidacloprid solution (100 ppm of active ingredient) and kept in a greenhouse under natural light conditions and at a temperature of $23 \pm 4^{\circ} \mathrm{C}$

Characterization of phloem-pd features produced by R. padi. Studies of EPG recordings on $R$. padi by our research team revealed the occurrence of intracellular punctures similar to the phloem-pd activity previously reported for $M$. persicae, such as lower frequency of intervals (distance between two downpeaks) in subphase II- 2 of the pd and lower pd magnitude compared with the standard-pds (Jiménez et al. 2018) (Fig. 1A and B). In contrast to those observed for $M$. persicae, two distinct patterns presumably occurring in phloem tissues were observed by $R$. padi before the E1 waveform. A specific intracellular pattern produced by $R$. padi (phloem-pd) showed similar characteristics to the phloem-pd reported for $M$. persicae, and an additional intracellular pattern with a longer subphase II-3, showing an E1-like pattern (called short-E1) (Fig. 1A and B) was observed. Therefore, in the present work we aimed to characterize both types of intracellular stylet punctures produced by $R$. padi by measuring the total duration of the $\mathrm{pd}$, the pd magnitude, the total number of intervals, and the frequency of intervals in the subphase II-2. All these distinct intracellular patterns were compared with the standard-pds and the subsequent E1 waveform. As opposed to $M$. persicae, R. padi often produced a short-E1 pattern, with a distinct II-2 subphase before the E1 phase (Fig. 1B). In fact, in previous studies a pd-like pattern (with distinct II-1 and II-2 subphases) occurring just before E1 waveform was also observed for other aphid species such as Aphis gossypii (Garzo et al. 2018). Therefore, we studied the duration of II- 2 subphase, the number and frequency of intervals, and other features of the intracellular stylet punctures produced by $R$. padi, including the II-2-like patterns observed within the E1 waveform. All variables were measured according to the methods described in Jiménez et al. (2018). A total of 51 EPG recordings were used to study and compare the distinctive features of the standard-pd, phloem-pd, short-E1, and E1 waveforms.

EPG setup. The feeding behavior of viruliferous $R$. padi individuals was monitored via EPG technique (Tjallingii 1978) in the laboratory with an average temperature of $24 \pm 1^{\circ} \mathrm{C}$. A direct current EPG device connected to an analog-to-digital converter was used. Viruliferous $R$. padi adults were collected from the colony and immediately attached to a gold wire $(18.5 \mu \mathrm{m}$ diameter) of an insect electrode with water-based silver glue. The same glue was used to glue the opposite end of the gold wire to a 3-cm-long copper wire $(0.2 \mathrm{~mm}$ diameter $)$ that was soldered to a brass nail $(1.2 \mathrm{~mm}$ diameter) with flux enhancement. The electrode nail was then inserted into the input connector of the first-stage amplifier (EPG probe). Substrate voltage was applied to the soil of the potted plants with a copper post (10 cm long, $2 \mathrm{~mm}$ diameter). Recordings were made with aphids feeding on the abaxial side of the single unfolded barley leaf. Signals were acquired and analyzed in Stylet+a software for Windows.

$R$. padi stylet activities associated with the inoculation of BYDV. To study the aphid stylets activities involved in successful inoculation of BYDV, the EPG signals produced by the viruliferous 
aphids were visually observed through a PC monitor, and recordings were artificially terminated by removing aphids with a paintbrush touch after the observation of different combinations of EPG activities, generating five different treatments: I, C waveform $+\geq 10$ standard-pds; II, C waveform + standard-pds $+\geq 1$ phloem-pds; III, C waveform + standard pds $+\geq 1$ phloem-pds $+\geq 1$ short-E1s; IV, C waveform + standard pds $+\geq 1$ phloem-pds + a single sieve element continuous salivation phase (E1 waveform); and V, C waveform + standard pds $+\geq 1$ phloem-pds $+\geq 1$ short-E1s + a single E1 phase (for a general view of the different EPG activities, see Fig. 1).

Aphids were removed from the test plants in which the feeding process was monitored by EPG (plant A). In plant A, the duration of inoculation access period (IAP) was determined by the observation of one of the different EPG signals detailed in the five treatments indicated in previous paragraph. Aphids were removed from plant A and individually transferred to a second test plant (plant B) for an IAP of $24 \mathrm{~h}$ to check the initial viruliferous condition of the aphid. BYDV transmission efficiency was calculated by dividing the total number of receptor plants that became infected during the aphid probing monitoring (only infected plant $\mathrm{A}$, or both infected plants $\mathrm{A}$ and $\mathrm{B}$ ) by the total number of plants in which the aphid was able to inoculate the virus (only infected plants $\mathrm{A}$, both $\mathrm{A}$ and $\mathrm{B}$, and only infected plants B). Aphids that were unable to inoculate the virus in any of the two test plants (both noninfected plants A and B) were discarded for the analysis. Each replicate was conducted with a different plant and aphid per EPG recording. The minimum number of replicates was done for treatment IV (21 replicates) and the maximum for treatment II (47 replicates) (Table 1).

A

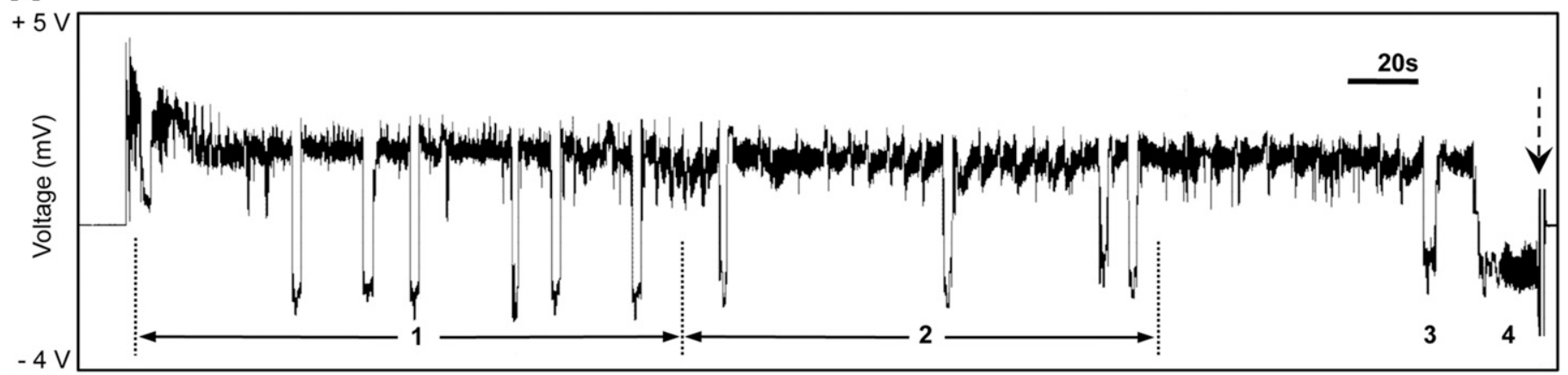

B
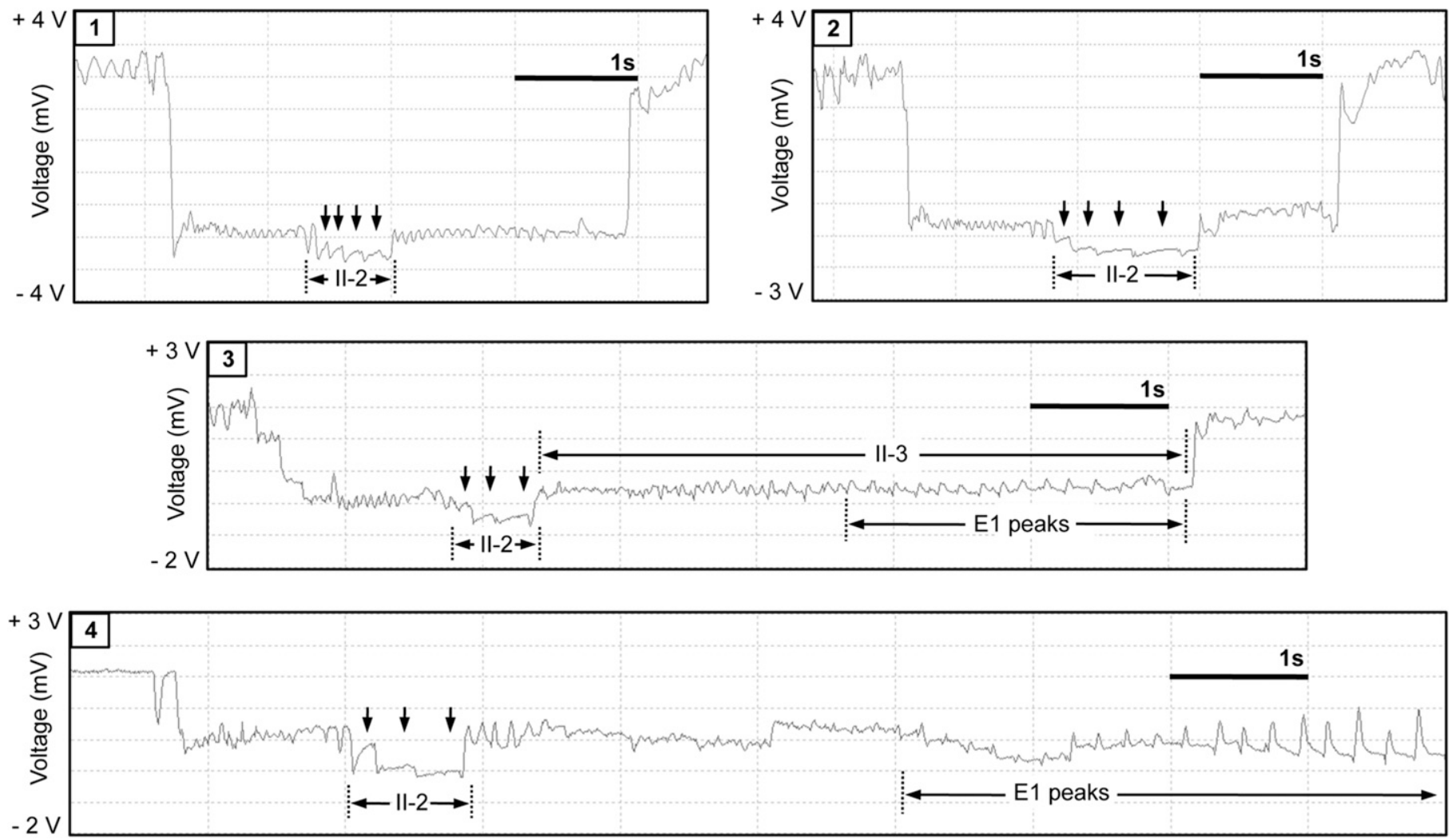

Fig. 1. A, General view of a standard EPG recording produced by viruliferous Rhopalosiphum padi in Hordeum vulgare artificially ended after the observation of a single sieve element salivation phase (E1 waveform). Down arrow indicates the exact point where the recording was artificially terminated. Numbers along the recording indicate the types of potential drops produced by the aphid. B, View of the different intracellular activities performed by $R$. padi in barley: 1, standard intracellular puncture (standard potential drop [standard-pd]); 2, intracellular puncture in phloem (phloem potential drop [phloem-pd]); 3, short salivation phase into sieve elements (short-E1); and 4, sieve element continuous salivation phase (E1 waveform). The short-E1 is particularly distinguished from previous phloempds and subsequent E1 waveform because of the occurrence of E1 peaks in the subphase II-3. Down arrows refer to each interval (distance between two downward peaks) in the subphase II-2. 
Comparison of the inoculation process between semipersistently and persistently transmitted, phloem-limited viruses. Recordings showing EPG activities that resulted in successful BYDV inoculation were thoroughly inspected in order to establish a relationship between the duration of aphid intrusion into phloem cells (activities related mainly to salivation in the phloem tissues) and successful BYDV infection in barley test plants. Any intracellular pattern produced by $R$. padi resulting in BYDV infection in the test plant was then considered phloem intrusion (phloem-pd, short-E1, and $\mathrm{E} 1$ waveform), and its duration was regressed to the transmission rate of BYDV obtained. Therefore, the total duration of the phloempds, short-E1s, and the E1 waveforms was calculated for each replicate within treatments II, III, IV, and V. A series of aphid salivation time intervals in phloem tissues were arbitrarily established: 0 to 4 s, 4 to $10 \mathrm{~s}, 10$ to $16 \mathrm{~s}, 16$ to $25 \mathrm{~s}, 25$ to $45 \mathrm{~s}$, and 45 to $90 \mathrm{~s}$. Such time intervals were regressed and plotted against the transmission efficiency of BYDV by $R$. padi.

For comparison of the transmission process of a semipersistently transmitted phloem-limited virus, we calculated the duration of aphid stylet intrusion in phloem cells from our previous reported study focused on the inoculation of BYV (Closterovirus) by M. persicae (Jiménez et al. 2018). We studied the EPG recordings performed for the BYV inoculation experiments to investigate the relationship between time of phloem intrusion and successful BYV inoculation. In this case, we calculated and added up the duration of the phloem-pds, the E1 waveform, and the transition phases between E1 and E2. The duration of aphid salivation in phloem cells and its relationship with the transmission efficiency of BYV by $M$. persicae were regressed in a similar way as previously described for BYDV.

Statistical analysis. Transmission rates of BYDV obtained in the different EPG treatments were compared by a Monte Carlo $\chi^{2}$ Pearson test, with Bonferroni correction (IBM Corp. 2017). Differences between the efficiency of BYV and BYDV transmission as a function of aphid salivation time in phloem tissues were analyzed by fitting a linear model for the data of each virus and comparing both linear models via a likelihood ratio test (R package nlme; Pinheiro et al. 2019). Models included an autoregressive variance-covariance matrix to account for time dependency in the measures of the efficiency of aphid transmission. Statistical analyses were conducted in $\mathrm{R}$ version 3.5.2 ( $\mathrm{R}$ Core Team 2018). The total duration of the intracellular puncture, the pd magnitude, the duration of subphase II2 , the number of intervals in subphase II-2, and the frequency of intervals were compared via a nonparametric Kruskal-Wallis test in the SPSS version 25.0 package (IBM Corp. 2017). A nonparametric test was performed when the variables showed a non-Gaussian distribution after the transformations ' $\ln (\mathrm{x}+1)$ ' or 'sqrt $(\mathrm{x}+1)$ ' were applied and checked for normality via Shapiro-Wilk $W$ test.

\section{RESULTS}

Phloem-pd features of $R$. padi: the phloem-pd and the short-E1. Results showed that the phloem-pd waveform recently reported for $M$. persicae was also produced by $R$. padi, showing similar electrical properties to the intracellular stylet punctures in the sieve element/companion cell complex (Jiménez et al. 2019). However, $R$. padi also produced an extra intracellular pattern showing an elongated II-3 subphase with characteristic positive peaks similar to those produced during the E1 waveform. This particular pattern observed during the II-3 subphase was not observed in the phloem-pd described for M. persicae. Therefore, we named this type of intracellular pattern "short-E1" because of its resemblance to the E1 waveform. The short-E1 was considered a distinct type of long pd because it exhibited a distinct II-1 and II-2 subphase but with an extended II-3 subphase with positive peaks very similar to those of the E1 waveform.

In terms of total duration, the short-E1 produced by $R$. padi showed longer total duration $(6.28 \pm 0.29 \mathrm{~s}$, ranging from 4.15 to $10.67 \mathrm{~s})$ than both the phloem-pd $(3.50 \pm 0.08 \mathrm{~s})(k=-59.76 ; P<$ $0.000)$ and the standard-pd $(3.76 \pm 0.09 \mathrm{~s})(k=-49.69 ; P<0.001)$. No differences in duration were observed between the standard and phloem-pd $(k=10.08 ; P=0.173)$ (Fig. 2 A) of $R$. padi. The average duration of the E1 waveform was $30.7 \mathrm{~s}$ (ranging from 16.9 to $60.9 \mathrm{~s}$ ), much longer than the average duration of the novel short-E1 reported in this work.

The potential drop magnitude of the E1 waveform $(2,152.73 \pm$ $172.3 \mathrm{mV}$ ) was similar to the drop observed in the phloem-pd $(2,673.90 \pm 203.37 \mathrm{mV})(k=18.95 ; P=0.078)$ and the short-E1 $(2,041.83 \pm 260.75 \mathrm{mV})(k=-5.07 ; P=0.640)$. The pd magnitude measured in the standard-pd $(3,014.18 \pm 205.09 \mathrm{mV})$ was significantly higher than in the short-E1 $(k=32.47 ; P=0.003)$ and the E1 waveform $(k=27.40 ; P=0.003)$. However, no differences in drop voltage magnitude were observed between the standard-pd and phloem-pd $(k=8.45 ; P=0.432)$ (Fig. 2B).

With regard to the duration of the subphase II-2, the standard-pd showed a shorter duration $(0.57 \pm 0.02 \mathrm{~s})$ in comparison with that of the phloem-pd $(0.81 \pm 0.05 \mathrm{~s})(k=-44.36 ; P<0.001)$, the short-E1 $(0.79 \pm 0.06 \mathrm{~s})(k=-37.63 ; P<0.001)$, and the E1 $(0.79 \pm 0.05 \mathrm{~s})(k=$ $-42.04 ; P<0.001)$. No differences in subphase II- 2 were observed between the phloem-pd and the short-E1 $(k=6.73 ; P=0.543)$, the phloem-pd and E1 $(k=2.32 ; P=0.826)$, and between the short-E1 and the E1 waveform $(k=-4.41 ; P=0.677)$ (Fig. 2C). In EPG recordings terminated during the $\mathrm{E} 1$ waveform, subphase II-2 was detected in 37 of 51 recordings. In the other 14 recordings, the E1 waveform followed the patterns of those produced by $M$. persicae, with no subphase II-2 before the typical E1 peaks.

Within patterns produced by $R$. padi in subphase II-2, the number of intervals in subphase II- 2 of the E1 waveform $(2.97 \pm 0.17$ Int $)$ was significantly lower in comparison with the standard-pd $(3.76 \pm 0.12$ Int $)(k=31.97 ; P<0.001)$, the phloem-pd (3.83 \pm 0.19 Int $)$ $(k=32.65 ; P<0.001)$, and the short-E1 (3.76 $\pm 0.21 \mathrm{Int})(k=29.37$; $P=0.003)$. The number of intervals observed in the standard-pd, phloem-pd, and short-E1 were well conserved in the three pds studied, with no significant differences $(k=-0.68, P=0.942$, for standard-pd and phloem-pd comparison $(k=2.61, P=0.780)$ for

TABLE 1. Transmission of Barley yellow dwarf virus (BYDV) by viruliferous Rhopalosiphum padi related to different stylet activities in Hordeum vulgare

\begin{tabular}{|c|c|c|c|c|c|}
\hline \multirow[b]{2}{*}{ EPG treatments } & \multirow[b]{2}{*}{ Plants $\mathrm{A}^{-} \mathrm{B}^{-}$} & \multicolumn{2}{|c|}{ Infected plants } & \multirow{2}{*}{$\begin{array}{c}\text { Proportion } \\
\mathrm{A}^{+} /\left(\mathrm{A}^{+}+\mathrm{B}^{+}\right)\end{array}$} & \multirow{2}{*}{$\begin{array}{l}\text { BYDV transmission } \\
\text { efficiency }\end{array}$} \\
\hline & & $\left(\mathrm{A}^{+} \mathrm{B}^{+}+\mathrm{A}^{+} \mathrm{B}^{-}\right)^{\mathrm{y}}$ & $\mathrm{B}^{+}$ & & \\
\hline I, Pathway $+\geq 10$ standard-pds & 2 & 0 & 42 & $0 / 42$ & $0 \mathrm{a}$ \\
\hline II, Pathway + standard-pds $+\geq 1$ phloem-pd(s) & 4 & 7 & 40 & $7 / 47$ & $14.9 \mathrm{ab}$ \\
\hline IV, Pathway + standard-pds $+\geq 1$ phloem-pds + a single E1 waveform & 0 & 5 & 16 & $5 / 21$ & $23.8 \mathrm{bc}$ \\
\hline \multicolumn{6}{|l|}{ V, Pathway + standard-pds $+\geq 1$ phloem-pds $+\geq 1$ short-E1s + a single } \\
\hline E1 waveform & 0 & 17 & 13 & $17 / 30$ & $56.7 \mathrm{c}$ \\
\hline
\end{tabular}

$\mathrm{y}$ The plant designated as plant A was the plant where the aphid feeding was monitored until production of the five waveform patterns was detected. The plant designated as plant $\mathrm{B}$ was the plant to which aphids were transferred from plant $\mathrm{A}$ in order to assess the initial virus acquisition by the aphid.

$\mathrm{z}$ Virus transmission efficiency (\%) was calculated by dividing the number of recordings where plant A became infected by the total number of recordings where plant A, plant B, or both test plants became infected. Different letters show significant differences according to a Monte Carlo $\chi^{2}$ test (Bonferroni correction) $(P>0.05)$. EPG, electrical penetration graph. 
standard-pd versus E1 $(k=3.28, P=0.752)$ for phloem-pd versus the short-E1 (Fig. 2D). The significantly shorter duration of subphase II-2 observed in the standard-pd resulted in a significantly higher frequency of intervals (Int/s) in this type of pd (6.83 $\pm 0.17 \mathrm{Int} / \mathrm{s})$ in comparison with the phloem-pd (4.85 $\pm 0.13 \mathrm{Int} / \mathrm{s})(k=61.00$; $P<0.001)$, the short-E1 (4.97 $\pm 0.15 \mathrm{Int} / \mathrm{s})(k=55.04 ; P<0.001)$, and the E1 waveform $(3.88 \pm 0.13 \mathrm{Int} / \mathrm{s})(k=93.29: P<0.001)$ (Fig. 2E). Interestingly, no differences in the frequency of intervals in subphase II-2 were observed between the phloem-pd and the short-E1 $(k=-5.97 ; P=0.589)$. However, the frequency of intervals observed in the E1 waveform was shorter than the ones observed in the phloem$\operatorname{pd}(k=32.29 ; P<0.002)$ and the short-E1 $(k=38.25 ; P<0.001)$.

In the $51 \mathrm{EPG}$ recordings terminated during the E1 phase $(n=21$ for treatment IV and $n=30$ for treatment V; Table 1), the E1 waveform was preceded by one of the two pd activities related with a phloem contact ( $n=21$ only showed the phloem-pd, $n=20$ only showed the short-E1) or by both activities, the phloem-pd and short-E1 patterns $(n=10)$. In recordings that showed both phloempd and short-E1, the short-E1 appeared always after the occurrence of the phloem-pd and preceding the E1 waveform (Fig. 1). A flow diagram with the sequence of intracellular punctures produced by $R$. padi before the E1 waveform is shown in Figure $3 \mathrm{~A}$ and $\mathrm{B}$.

$R$. padi stylet activities associated with BYDV inoculation. No BYDV transmission was observed when aphids were allowed to produce standard intracellular punctures only ( 0 of 42 replicates) (Table 1). Transmission tests showed that BYDV inoculation occurred at a low rate after the production of a phloem-pd (7 of 47 replicates; $14.9 \%$ ). Interestingly, BYDV inoculation efficiency was doubled when $R$. padi was allowed to produce a short-E1 before the occurrence of the E1 waveform (13 of $39 ; 33.3 \%)$.

Within recordings terminated during the first E1 phase, aphids performing a short-E1 (and also phloem-pds) before the E1 waveform doubled the BYDV inoculation rate (17 of $30 ; 56.7 \%$ ) in comparison with aphid that performed only a single phloem-pd but no short-E1 ( 5 of $21 ; 23.8 \%$ ), but with no significant differences between treatments (Table 1).

Relationship between the duration of intracellular intrusion of aphid stylets in phloem cells and the transmission of phloemlimited viruses. Linear models used to compare the mode of transmission of two phloem-limited viruses transmitted in a semipersistent (BYV) or persistent manner (BYDV) as a function of time of aphid salivation in the phloem cells showed differences in the process of transmission of both virus species $(P<0.0001)$. The transmission efficiency obtained for BYV was higher than BYDV at all the periods of phloem salivation times established, reaching the highest BYV efficiency at shorter times of aphid salivation. Whereas the inoculation efficiency of BYV reached the highest value after $4 \mathrm{~s}$ of aphid salivation into the SE-CC complex (6 of 6; $100 \%$ ), the inoculation efficiency of BYDV was only $10.7 \%$ (3 of 28 ) (Fig. 4). In the period of 4 to $10 \mathrm{~s}$ of aphid salivation, BYV inoculation efficiency dropped to $70 \%$ (7 of 10 ), whereas BYDV transmission slightly increased to $28.1 \%$ (9 of 32). BYV transmission rates followed the same trend after a period of aphid salivation of 10 to $16 \mathrm{~s}(60 \%), 16$ to $25 \mathrm{~s}(75 \%), 25$ to $45 \mathrm{~s}(59.1 \%)$, and 45 to $90 \mathrm{~s}(75 \%)$, with BYDV less efficiently transmitted than BYV in each time period studied (Fig. 4).

\section{DISCUSSION}

Several studies focused on the transmission mechanism of BYDV by monitoring the feeding process of its main aphid vectors S. avenae and R. padi (Prado and Tjallingii 1994; Scheller and Shukle 1986; Schliephake et al. 2013). Nevertheless, our work is the first to show specific aphid stylet activities in the plant involved in the early steps of inoculation of a persistently transmitted, phloem-limited virus. Our main finding is that BYDV transmission can occur during brief intracellular punctures in SE and CC before

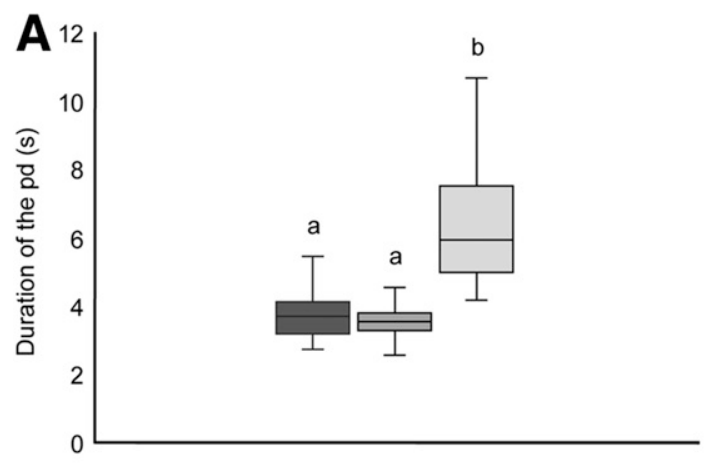

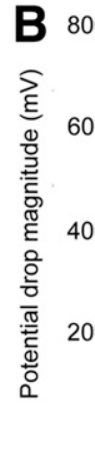
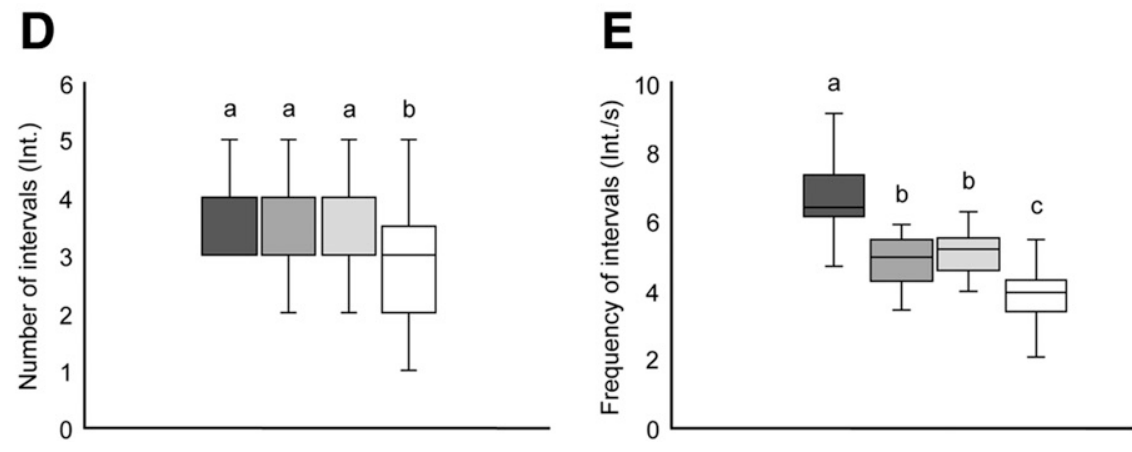

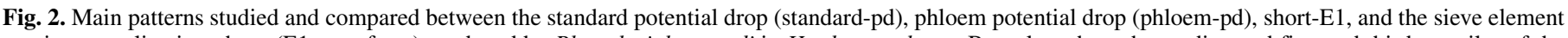

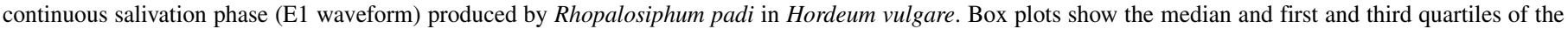

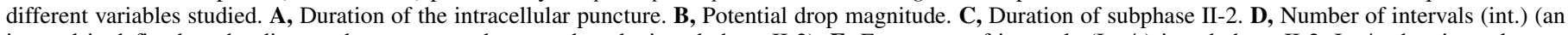

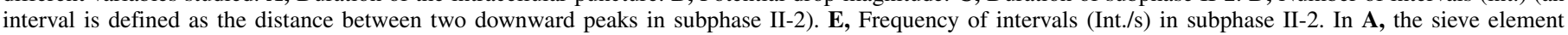

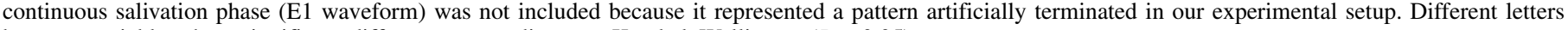
between variables show significant differences according to a Kruskal-Wallis test $(P<0.05)$. 
the continuous salivation phase in the phloem sieve elements (E1 waveform). Furthermore, the phloem-pd produced by $R$. padi in barley was easily distinguished from other intracellular punctures produced in nonvascular tissues (standard-pds) by a direct current EPG device, as previously reported for $M$. persicae in sugar beet (Jiménez et al. 2018). The phloem-pd waveform observed for $R$. padi had very similar properties to the one already described for $M$. persicae, showing lower pd magnitude and a lower frequency of intervals in the subphase II- 2 than the standard pd. However, the newly characterized short-E1 pattern had intermediate characteristics between a phloem-pd and the E1 waveform, with a longer II-3 subphase showing the characteristic positive peaks of E1 (Prado

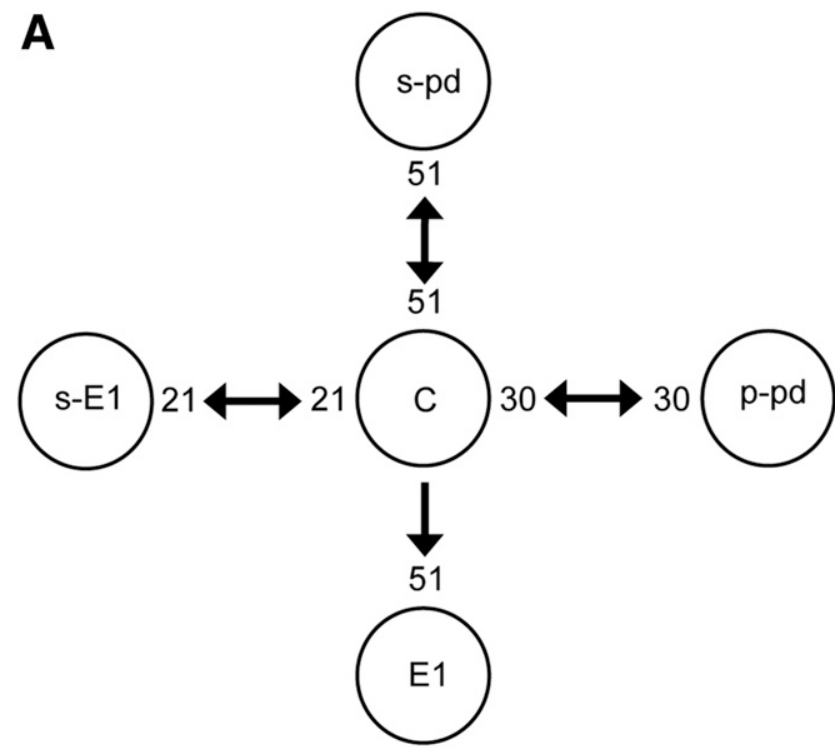

B

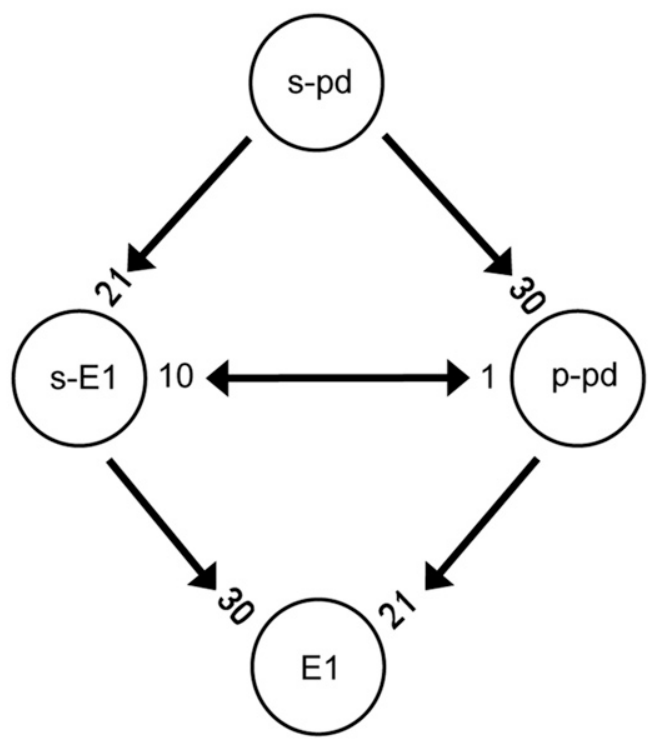

Fig. 3. A, Flow diagram showing the sequence of intracellular punctures produced by viruliferous Rhopalosiphum padi in Hordeum vulgare before the phloem sieve element phase of the aphid (E1 waveform). B, Sequence of intracellular punctures observed before production of the sieve element continuous salivation phase (E1 waveform) of the aphid. Electrical penetration graph recordings artificially ended after the observation of the E1 waveform were studied $(n=51)$. Values close to arrowheads indicate the total number of recordings where a certain waveform was followed by any other. C, waveform $\mathrm{C}$, in which $\mathrm{G}$ and $\mathrm{F}$ waveforms produced by $R$. padi individuals were included; p-pd, phloem-pd; s-E1, short aphid salivation in sieve elements; E1, sieve element continuous salivation phase; s-pd, standard-pd. and Tjallingii 1994). Both phloem-pd and short-E1 activities are probably occurring in phloem tissues because of the results we obtained in the EPG-assisted BYDV inoculation experiments and the phloem-limited condition of BYDV in their host plants (Gray and Gildow 2003).

In terms of BYDV inoculation efficiencies in association with the different EPG patterns produced by $R$. padi, the findings of this work are strongly in agreement with those obtained by Prado and Tjallingii (1994). Results obtained in this work demonstrated the hypothesis already stated by Prado and Tjallingii regarding the presumable intrusions of stylets into phloem cells before the E1 waveform as responsible for few aphids being able to inoculate BYDV. However, in our study we could associate the transmission of BYDV before the E1 waveforms to two different intracellular punctures: phloem-pd and short-E1 produced by $R$. padi, presumably in phloem cells. In fact, they obtained $12.90 \%$ (4 of 31) of BYDV inoculation rate in the treatment "pathway," which included the production of different types of potential drops (probably also phloem-pds and short-E1s). More precisely, the "pathway" treatment that Prado and Tjallingii used for their study would correspond with treatments I + II + III of our experimental procedure, resulting in a similar BYDV inoculation efficiency of $15.6 \%$ (20 of 128) $\left(\chi^{2}=\right.$ $0.144 ; P=0.704)$. Despite the fact that no E1 phase was recorded during the treatment "pathway," the occurrence of both phloem-pds and short-E1 within those recordings was quite likely. Here we could clearly associate the primarily inoculation of BYDV to the phloem-pd and to a short-E1, discarding the possibility that BYDV inoculation can occur when only standard-pds (stylet penetrations in nonvascular cells) are produced by the aphid.

On the other hand, Scheller and Shukle (1986) clearly associated the inoculation of BYDV (MAV and PAV isolates) with phloem contacts visualized by the X-waveform followed by the sap phloem Ip produced by $S$. avenae in oat (Avena sativa) when an alternating current EPG amplifier was used. Histological preparations positively associated the X-waveform with aphid stylet contacts with phloem cells, with no identification of the type of phloem cell contacted by the stylets. The X-waveform always appeared before the ingestion from the phloem (Ip) and probably represents the same activities as those of phloem-pd or short-E1 that we observed for $R$. padi when using our direct current EPG device. Furthermore, phloem-pd here described for $R$. padi and previously reported for $M$. persicae also occurred in S. avenae (J. Jiménez, unpublished data). However, detailed analysis of the phloem-pd activity produced by $S$. avenae needs further investigation. Transmission of another semipersistently transmitted virus, Maize chlorotic dwarf virus (MCDV; Waikavirus), was also observed only when X-waveform was produced by the leafhopper Graminella nigrifrons (Wayadande and Nault 1993). A positive association between longer X-waveforms and higher transmission of MCDV was also observed.

The analysis of EPG recordings and characterization of the phloem-pd and short-E1 produced by $R$. padi confirmed that a short IAP is needed for BYDV transmission when $R$. padi is used as a vector. Power et al. (1991) reported a BYDV inoculation efficiency of 47.1 and $58.6 \%$ (for PAV and RPV isolates, respectively) after an IAP of $2 \mathrm{~h}$. The analysis of our EPG recordings artificially terminated during the $\mathrm{E} 1$ phase revealed that $R$. padi reached the E1 phase in a mean time of $54.5 \mathrm{~min}$ from the onset of stylet penetration in barley tissues (waveform C), resulting in a BYDV inoculation rate of $56.7 \%$. Nevertheless, Power et al.'s work also reported fairly high BYDV inoculation efficiency after $30 \mathrm{~min}$ as an IAP (24.6 and $33.3 \%$ for PAV and RPV isolate, respectively). In fact, here we showed that $R$. padi can briefly penetrate phloem cells and therefore transmit BYDV in a short period of time, much faster than previously thought. According to our study, the minimum time threshold needed for stylet penetration of phloem cells was 4.6, 6.4, and 7.3 min for a phloem-pd, short-E1, and E1 waveform, respectively (data not shown).

As shown in Figure 1B, subphase II-3 of the short-E1 waveform shows positive peaks similar to those observed during E1, suggesting 
that the same feeding activity occurs. Therefore, the elongated phase of the II-3 subphase within the short-E1 probably represents watery salivation into the sieve elements, as shown for the E1 waveform (Prado and Tjallingii 1994). Conversely, as previously shown, the likely activity occurring during the II-3 subphase of the standard-pds is ingestion of cell contents and thus acquisition of virus particles transmitted in a nonpersistent manner (Collar and Fereres 1998; Martín et al. 1997). Furthermore, we found a close relationship between the production of the short-E1 waveform and inoculation success of BYDV. Aphids that performed a short-E1 before the E1 phase doubled BYDV transmission efficiency (17 of $30 ; 56.7 \%$ ) in comparison with the aphids that failed to make a short-E1 and produced a phloem-pd before the E1 waveform (5 of $21 ; 23.8 \%$ ). Particularly in our work, aphids that combined both a phloem-pd and a short-E1 before the E1 phase resulted in a very high inoculation rate $(9$ of $10 ; 90 \%)$. The positive relationship between number of stylet penetrations into the phloem and increased BYDV inoculation rate was previously reported by Scheller and Shukle (1986).

The differences in pd voltage between standard-pds, phloem-pds and short-E1 are more intriguing than those previously reported for $M$. persicae. In case of $R$. padi, a gradual decrease in pd magnitude is observed from the standard-pds until the phloem phase is reached (Fig. 1A). In fact, the paucity of plasmodesmata connections between the phloem parenchyma cells and the thin-walled SEs (and adjoining CCs) and thick-walled SEs supports the hypothesis of an apoplastic pathway (Evert et al. 1996). That could result in a relative isolation of both thin-walled SEs and CCs and thick-walled SEs complexes in barley, as observed for other plant species after an apoplastic phloem loading (van Bel and van Rijen 1993). However, it is not possible to determine what type of phloem cell is being punctured during both phloem-pd and short-E1 based exclusively on the magnitude of the pd. Our initial hypothesis postulates that the phloem-pd could represent stylet intrusions likely in the thin-walled SEs and CCs or thick-walled SE complex. However, the short-E1 may represent a stylet intrusion exclusively in SEs. In aphids producing both phloem-pd and a short-E1, the short-E1 was always produced just after the phloem-pd and before the E1 waveform with an elapsed time of $11.4 \mathrm{~s}$. Therefore, aphid stylets remain in the same cell with almost no change in the stylet tip position, with the same sieve element being punctured by the aphid during the short-E1 and the E1 waveform. A similar phenomenon of the same sieve element being punctured consecutively several times was reported for Brevicoryne brassicae and some other aphid species and was named repetitive potential drops (Tjallingii and Gabrys 1999).

Both noncirculative (BYV) and circulatively transmitted (BYDV) viruses are transmitted during brief intracellular punctures produced by aphids in the phloem tissues of the host plant. However, our results reflected the different mode of transmission between phloem-limited viruses transmitted in a semipersistent or persistent manner by aphids. The main difference between the mechanism of transmission between a semipersistent cuticle-borne aphid-transmitted virus (e.g., BYV) and a circulative saliva-borne persistently transmitted virus (e.g., BYDV) is that BYDV transmission efficiency is directly related to the duration of stylet intrusions (salivation) in phloem cells, whereas that of BYV is not (Fig. 4). Conversely, ejection of BYV particles is driven by single intracellular stylet penetrations in phloem cells that result in very high inoculation efficiency. Therefore, in the case of the semipersistent, cuticula-borne BYV, some specific aphid activity is probably mediating the virus particles out of the maxillary stylets, suggesting that the inoculation of this virus is independent of the time spent by the aphid in salivation activities in phloem cells. In fact, a single very brief intracellular puncture (of about $4 \mathrm{~s}$ ) in the SE-CC complex produced by viruliferous $M$. persicae was enough to inoculate BYV at a very high transmission rate (100\%) to sugar beet. Thus, BYV is rapidly dislodged from its specific retention sites in the cuticle (either the common duct of the maxillary stylets or the foregut) and readily

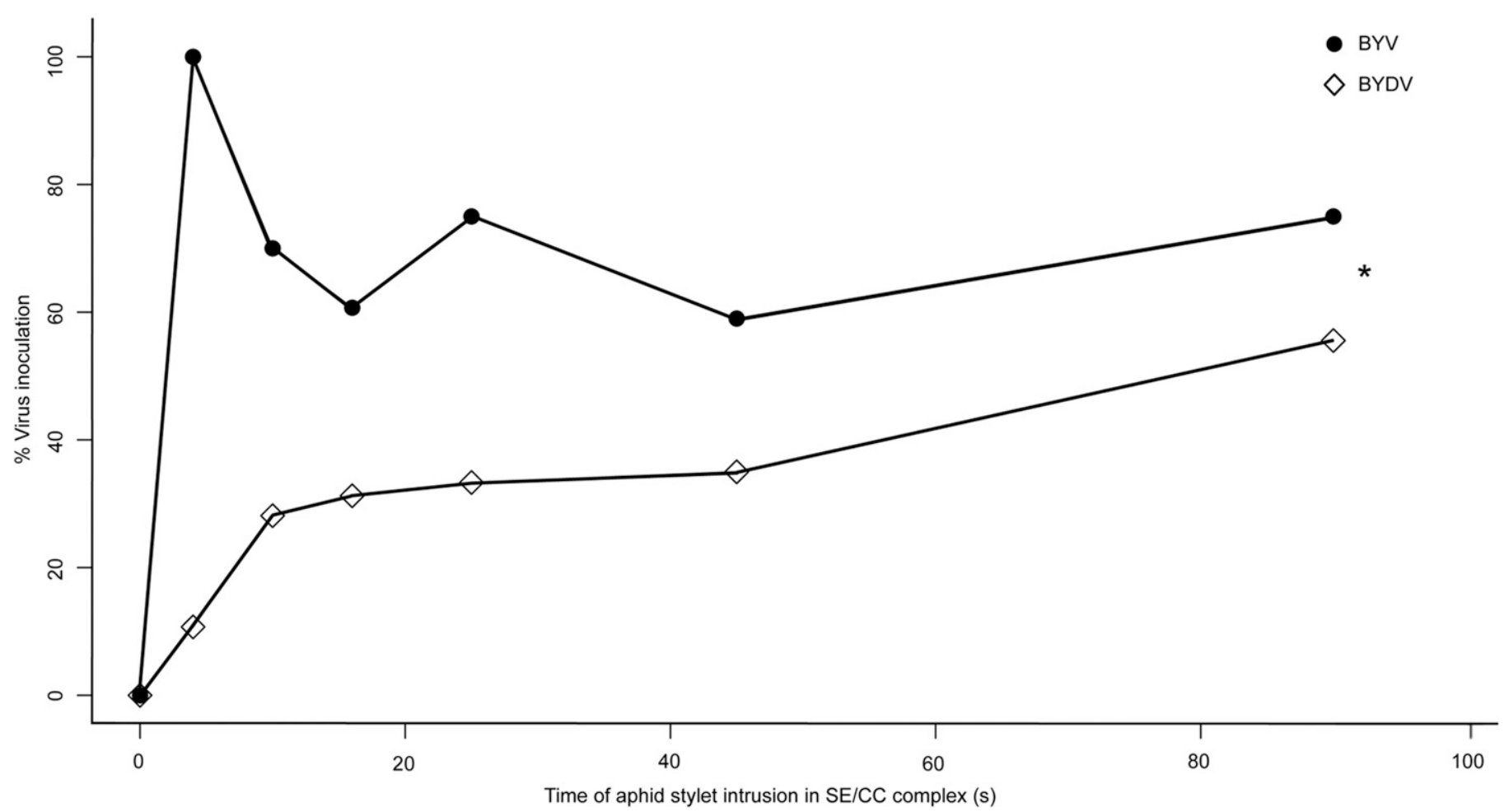

Fig. 4. Comparison of the relationship between total time of salivation in phloem tissues by Rhopalosiphum padi and Myzus persicae and successful inoculation of Barley yellow dwarf virus (BYDV) and Beet yellows virus (BYV), respectively. Times of aphid stylet penetration in phloem were classified in different periods: 0 to $4 \mathrm{~s}, 4$ to $10 \mathrm{~s}, 10$ to $16 \mathrm{~s}, 16$ to $25 \mathrm{~s}, 25$ to $45 \mathrm{~s}$, and 45 to $90 \mathrm{~s}$. *Asterisk indicates significant differences between BYV and BYDV inoculation efficiencies in the period of time studied according to a linear model comparison by a likelihood ratio test $(P<0.05)$. Data on M. persicae salivation in phloem in relation with BYV inoculation were derived from the study of the electrical penetration graph recordings included in Jiménez et al. (2018). 
inoculated into the plant. Previous studies aimed at localizing virions of semipersistently aphid-transmitted viruses in the vector mouthparts showed retention sites in the foregut of the vector (Killiny et al. 2016; Murant et al. 1976). Foregut attachment has also been shown for related whitefly-transmitted viruses of the same family as BYV (Chen et al. 2011). That suggests that BYV particles could also attach to the foregut of $M$. persicae. If so, the most likely mechanism for BYV inoculation would be egestion during brief intracellular punctures in phloem cells (now visualized and identified as phloem-pds). This transmission theory was proposed long ago by Harris (1977) as a mechanism for explaining the transmission of nonpersistent viruses. In the case of BYDV, the injection of virus particles into the plants is mediated solely by the watery saliva injected by the aphid into the plant cells from the accessory salivary glands (Gray and Gildow 2003). Also, in our study, the rate of BYDV inoculation rose proportionally with longer times of aphid salivation into phloem cells, that is to say, with the quantity of saliva ejected. As reported for Pea enation mosaic virus (PEMV; Enamovirus), ejection of watery saliva during subphase II-1 is the mechanism responsible for successful injection of circulative virus particles into the cells (Powell 2005), similar to what was earlier observed for viruses transmitted in a nonpersistent manner (Martín et al. 1997). Although watery saliva is secreted by the aphid from the onset of the first probe in case of phloem-limited viruses (Moreno et al. 2011), only stylet intrusions into phloem cells lead to systemic infection.

Undoubtedly, unknown factors are influencing BYDV inoculation, resulting in a less efficient process in comparison with the semipersistently transmitted BYV when the aphid salivates in phloem cells during the brief intracellular punctures before the E1 phase. The mechanisms underlying sudden BYV delivery into phloem cells also warrant future investigation. To confirm that BYV is transmitted by egestion of previously ingested phloem sap requires unequivocal localization and retention sites of virus particles in the foregut or the cibarium of its aphid vector. The findings reported here represent an important step forward to elucidate the transmission mechanisms of phloemlimited viruses.

\section{ACKNOWLEDGMENTS}

We thank María Plaza for aphid, virus, and plant maintenance; Israel Pagán for assistance with the statistical procedures; and Martin Godefroid and Laura Barrios for their advice on data analysis.

\section{LITERATURE CITED}

Blanc, S., and Michalakis, Y. 2016. Manipulation of hosts and vectors by plant viruses and impact of the environment. Curr. Opin. Insect Sci. 16:36-43.

Chen, A. Y. S., Walker, G. P., Carter, D., and Ng, J. C. K. 2011. A virus capsid component mediates virion retention and transmission by its insect vector. PNAS 108:16777-16782.

Chen, J. Q., Martin, B., Rahbé, Y., and Fereres, A. 1997. Early intracellular punctures by two aphid species on near-isogenic melon lines with and without the virus aphid transmission (Vat) resistance gene. Eur. J. Plant Pathol. 103:521-536.

Clark, M. F., and Adams, A. N. 1977. Characteristics of the microplate method of enzyme-linked immunosorbent assay for the detection of plant viruses. J. Gen. Virol. 34:475-483.

Collar, J. L., and Fereres, A. 1998. Nonpersistent virus transmission efficiency determined by aphid probing behavior during intracellular puncture. Environ. Entomol. 27:583-591.

D’Arcy, C. J. 1995. Symptomology and host range of barley yellow dwarf. Pages 9-28 in: Barley Yellow Dwarf: 40 Years of Progress. D. J. D’Arcy and P. A. Burnett, eds. American Phytopathological Society, St. Paul, MN.

Esau, K. 1957. Phloem degeneration in Graminae affected by the barley yellow-dwarf virus. Am. J. Bot. 44:245-251.

Esau, K. 1968. Viruses in Plant Hosts: Form, Distribution, and Pathologic Effects. University of Wisconsin Press, Madison, WI.

Evert, R. F., Russin, W. A., and Botha, C. E. J. 1996. Distribution and frequency of plasmodesmata in relation to photoassimilate pathway and phloem loading in the barley leaf. Planta 198:572-579.
Fereres, A. 2016. Aphid behavior and the transmission of noncirculative viruses. Pages 31-45 in: Vector-Mediated Transmission of Plant Pathogens. J. K. Brown, ed. American Phytopathological Society, St. Paul, MN.

Fereres, A., and Moreno, A. 2009. Behavioural aspects influencing plant virus transmission by homopteran insects. Virus Res. 141:158-168.

Fereres, A., and Raccah, B. 2015. Plant virus transmission by insects. In: eLS. John Wiley \& Sons, Inc, Chichester, UK.

Garret, A., Kerlan, C., and Thomas, D. 1996. Ultrastructural study of acquisition and retention of potato leafroll luteovirus in the alimentary canal of its aphid vector, Myzus persicae Sulz. Arch. Virol. 141: 1279-1292.

Garzo, E., Fernández-Pascual, M., Morcillo, C., Fereres, A., Gómez-Guillamón, M. L., and Tjallingii, W. F. 2018. Ultrastructure of compatible and incompatible interactions in phloem sieve elements during the stylet penetration by cotton aphids in melon. Int. J. Insect Sci. 25:631-642.

Gildow, F. E. 1990. Current status of barley yellow dwarf in the United Stated: a regional report. Pages 11-20 in: World Perspectives on Barley Yellow Dwarf. P. A. Burnet, ed. CIMMYT, Mexico.

Gildow, F. E. 1999. Luteovirus transmission and mechanisms regulation vector specificity. Pages 88-112 in: The Luteoviridae. H. G. Smith and H. Barker, eds. CAB International, Oxon, UK.

Gildow, F. E., and Gray, S. M. 1993. The aphid salivary gland basal lamina as a selective barrier associated with vector-specific transmission of Barley yellow dwarf virus. Phytopathology 83:1293-1302.

Gray, S. M., and Gildow, F. E. 2003. Luteovirus-aphid interactions. Annu. Rev. Phytopathol. 41:539-566.

Habekuß, A., Schliephake, E., Matthes, P., Hartled, H., Mehner, S., Grüntzig, M., and Fuchs, E. 2002. Zum Auftreten des Gerstengelbverzwergungsvirus und seiner Vektoren in Sachsen-Anhalt. Pages 111-114 in: 53. Tagung der Vereinigung de Pflanzenzüchter und Saatgutkaufleute.

Halbert, S. C., and Voegtlin, D. 1995. Biology and taxonomy of vectors of barley yellow dwarf viruses. Pages 217-258 in: Barley Yellow Dwarf: 40 Years of Progress. C. L. D'Arcy and P. A. Burnett, eds. American Phytopathological Society, St. Paul, MN.

Harris, K. F. 1977. An ingestion-egestion hypothesis of noncirculative virus transmission. Pages 165-220 in: Aphids as Virus Vectors. K. F. Harris and K. Maramorosch, eds. Academic Press, Cambridge, MA.

IBM Corp. 2017. IBM SPSS Statistics for Macintosh, Version 25.0. IBM Corp., Armonk, NY.

Ingwell, L. L., and Bosque-Pérez, N. A. 2015. The invasive weed Ventenata dubia is a host of Barley yellow dwarf virus with implications for an endangered grassland habitat. Weed Res. 55:62-70.

Ingwell, L. L., Zemetra, R., Mallory-Smith, C., and Bosque-Pérez, N. A. 2014. Arundo donax infection with Barley yellow dwarf virus has implications for biofuel production and non-managed habitats. Biomass Bioenergy $66: 426-433$

Jiménez, J., Garzo, E., Alba-Tercedor, J., Moreno, A., Fereres, A., and Walker, G. P. 2019. The phloem-pd: a distinctive brief sieve element stylet puncture prior to sieve element phase of aphid feeding behavior. Arthropod-Plant Interact. https://doi.org/10.1007/s11829-019-09708-w

Jiménez, J., Tjallingii, W. F., Moreno, A., and Fereres, A. 2018. Newly distinguished cell punctures associated with transmission of the semipersistent phloem-limited Beet yellows virus. J. Virol. 92:e01076-18.

Killiny, N., Harper, S. J., Alfaress, S., Mohtar, C. E., and Dawson, W. O. 2016. Minor coat and heat chock proteins are involved in the binding of Citrus tristeza virus to the foregut of its aphid vector, Toxoptera citricida. Appl. Environ. Microbiol. 82:6294-6302.

Martín, B., Collar, J. L., Tjallingii, W. F., and Fereres, A. 1997. Intracellular ingestion and salivation by aphids may cause the acquisition and inoculation of non-persistently transmitted plant viruses. J. Gen. Virol. 78: 2701-2705.

Mauck, K. E., Chesnais, Q., and Shapiro, L. R. 2018. Evolutionary determinants of host and vector manipulation by plant viruses. Adv. Virus Res. 101: 189-250.

Moreno, A., Garzo, E., Fernandez-Mata, G., Kassem, M., Aranda, M. A., and Fereres, A. 2011. Aphids secrete watery saliva into plant tissues from the onset of stylet penetration. Entomol. Exp. Appl. 139:145-153.

Moreno, A., Tjallingii, W. F., Fernandez-Mata, G., and Fereres, A. 2012. Differences in the mechanism of inoculation between a semi-persistent and a non-persistent aphid-transmitted plant virus. J. Gen. Virol. 93: 662-667.

Murant, A. F., Roberts, I. M., and Elnagar, S. 1976. Association of virus-like particles with the foregut of the aphid Cavariella aegopodii transmitting the semi-persistent viruses anthriscus yellows and parsnip yellow fleck. J. Gen. Virol. 31:47-57.

Palacios, I., Drucker, M., Blanc, S., Leite, S., Moreno, A., and Fereres, A. 2002. Cauliflower mosaic virus is preferentially acquired from the phloem by its aphid vectors. J. Gen. Virol. 83:3163-3171. 
Pinheiro, J., Bates, D., DebRoy, S., Sarkar, D., and R Core Team. 2019. nlme: Linear and Nonlinear Mixed Effects Models. R package version 3.1-140. https://CRAN.R-project.org/package=nlme

Powell, G. 2005. Intracellular salivation is the aphid activity associated with inoculation or non-persistently transmitted viruses. J. Gen. Virol. 86: 469-472.

Powell, G., Pirone, T., and Hardie, J. 1995. Aphid stylet activities during potyvirus acquisition from plants and an in vitro system that correlate with subsequent transmission. Eur. J. Plant Pathol. 101:411-420.

Power, A. G., Seaman, A. J., and Gray, S. M. 1991. Aphid transmission of Barley yellow dwarf virus: inoculation access periods and epidemiological implications. Phytopathology 81:545-548.

Prado, E., and Tjallingii, W. F. 1994. Aphid activities during sieve element punctures. Entomol. Exp. Appl. 72:157-165.

R Core Team. 2018. R: A Language and Environment for Statistical Computing. R Foundation for Statistical Computing, Vienna, Austria.

Scheller, H. V., and Shukle, R. H. 1986. Feeding behavior and transmission of Barley yellow dwarf virus by Sitobion avenae on oats. Entomol. Exp. Appl. 40:189-195.
Schliephake, E., Habekuss, A., Scholz, M., and Ordon, F. 2013. Barley yellow dwarf virus transmission and feeding behaviour of Rhopalosiphum padi on Hordeum bulbosum clones. Entomol. Exp. Appl. 146:347-356.

Tjallingii, W. F. 1978. Electronic recording of penetration behavior by aphids. Entomol. Exp. Appl. 24:721-730.

Tjallingii, W. F. 1985. Membrane potentials as an indicator for plant-cell penetration by aphid stylets. Entomol. Exp. Appl. 38:187-193.

Tjallingii, W. F. 1988. Electrical recording of stylet penetration activities. Pages 95-108 in: Aphids: Their Biology. Nature Enemies and Control, vol. 2B. A. K. Minks and P. J. Harrewijn, eds. Elsevier, Amsterdam.

Tjallingii, W. F., and Gabrys, B. 1999. Anomalous stylet punctures of phloem sieve elements by aphids. Entomol. Exp. Appl. 91:97-103.

van Bel, A. J. E., and van Rijen, H. V. M. 1993. Microelectrode-recorded development of the symplastic autonomy of the sieve elements/companion cells complex in the stem phloem of Lupinus luteus L. Planta 192: 165-175.

Wayadande, A. C., and Nault, L. R. 1993. Leafhopper probing behavior associated with Maize chlorotic dwarf virus transmission to maize. Phytopathology 83:522-526. 